\title{
ANÁLISE ESTRUTURAL EM COLHEDORA DE CANA
}

\author{
Rômulo Reis ${ }^{1}$, Gabriel Gueller ${ }^{2}$ e Mariana Batista ${ }^{3}$ \\ ${ }^{1}$ Smarttech Serviços e Engenharia \\ E-mails: romulo.reis@ @marttech.com.br, gabriel.gueller@smarttech.com.br, \\ mariana.batista@smarttech.com.br
}

\section{RESUMO}

A colheita mecanizada trouxe uma série de desafios para o agricultor e para os fabricantes de máquinas agrícolas. Do ponto de vista estrutural, a máquina deve ser suficientemente robusta para suportar todas as demandas inerentes ao seu uso. Para isso, a definição de cargas e condições de uso se premissas fundamentais para o desenvolvimento do produto. Assim, este trabalho tem como objetivo descrever as etapas e metodologia utilizadas para redefinição de um chassi de uma colhedora de cana-de-açúcar. Essas etapas vão da instrumentação no campo para a definição de cargas, através da análise de elementos finitos e otimização, e testes em laboratório para validação.

\section{INTRODUÇÃO}

Definição de critérios de projeto é um grande desafio para os desenvolvedores de máquinas agrícolas. O modo como o equipamento deve atuar a fim de cumprir seu desempenho funcional de colheita, manejo de solo e cultura é cuidadosamente planejado, porém do ponto de vista estrutural esses equipamentos ainda têm desafios a serem enfrentados. A diversidade de solos e culturas torna a determinação de esforços uma tarefa árdua. Sem o devido conhecimento dos esforços atuante na estrutura não é possível realizar um dimensionamento adequado, tornando os demais passos do desenvolvimento uma tentativa e erro baseado somente na experiência e intuição do projetista.

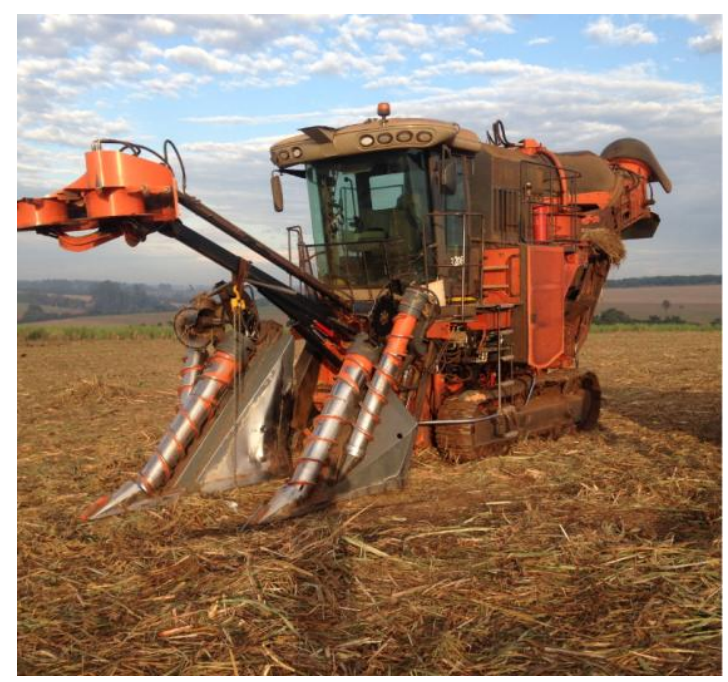

Figura 1: Colhedora Santal. 
Esse trabalho irá mostrar em detalhes a metodologia de engenharia aplicada ao desenvolvimento de uma colhedora de cana desde o levantamento de cargas em campo passando pelo modelo de elementos finitos e laboratório até a validação em campo.

\section{METODOLOGIA}

A colhedora base line apresentava trincas prematuras entre $10 \%$ a $15 \%$ da vida desejada. Oito trincas principais eram recorrentes e foram estratégicas para o trabalho para a definição das curvas de fadiga assim como para instrumentação.

- O primeiro passo é determinar os carregamentos. A maneira mais prática para isso é realizando uma instrumentando uma máquina já existente com extensômetros e acelerômetros.

- As regiões da máquina a serem instrumentadas são indicadas por uma simulação de elementos finitos prévia, que indica a localização e a direção mais adequada para a colagem dos extensômetro.

- Após a instrumentação, rotas e manobras específicas são definidas para serem medidas nos mais diferentes tipos e densidades de plantio de cana. Essa etapa é definida em conjunto com a equipe de pós-venda que identifica aplicações e manuseio das mais diferentes formas e severidades.

- Com os resultados de medição é realizada a calibração entre o modelo de elementos finitos. Esta etapa é crucial para a qualidade das etapas seguintes, pois todas as modificações e tentativas de melhoria serão realizadas no modelo virtual.

- Com o modelo calibrado e com os carregamentos bem estabelecidos se inicia a etapa de melhoria do produto para que atenda os critérios de tensão para o objetivo de vida estabelecido.

- Após se definir a geometria do produto um primeiro protótipo é construído e levado para laboratório para um teste acelerado. Um primeiro teste acelerado é realizado no chassi base line a fim de se reproduzir as mesmas falhas de campo validando assim um formato de teste acelerado comprove a simulação virtual e reproduza uma condição de uso real do produto.

- Todas as etapas anteriores levam a um protótipo mais próximo do produto final, porém não eliminam por completo a chance de falha, apenas aumentam a chance de sucesso. Por esse motivo uma instrumentação final se torna conveniente para validação do sucesso do produto. 


\section{INSTRUMENTAÇÃO INICIAL}

Antes da instrumentação um modelo de elementos finitos foi gerado para determinação dos pontos críticos Figura 2.

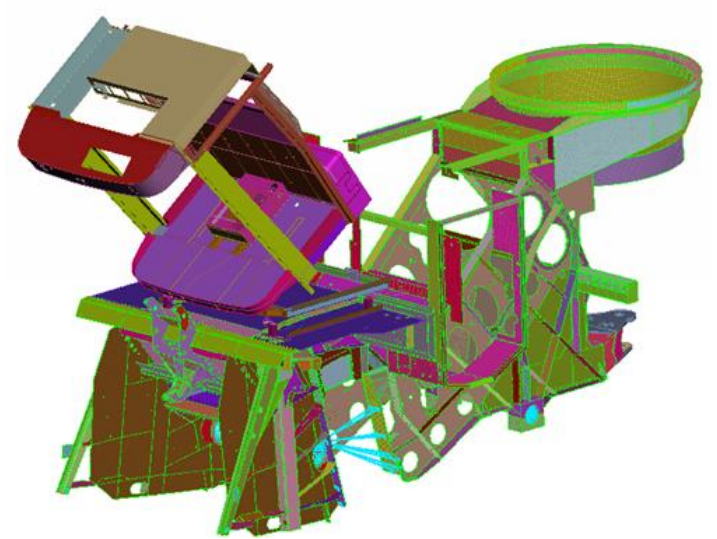

Figura 2: Modelo inicial.

As cargas utilizadas inicialmente possuíam valores genéricos, pois no momento a localização dos pontos críticos na estrutura e a direção da máxima tensão são mais importantes que sua magnitude. Quatro casos de carregamentos foram considerados inicialmente e são ilustrados na Figura 3:

- Carregamento vertical de gravidade 1 g estático (próprio peso).

- Carregamento lateral de gravidade $1 \mathrm{~g}$ (curva).

- Carregamento longitudinal de gravidade $1 \mathrm{~g}$ (frenagem).

- Carregamento de torção do chassi (deslocamento de 10 milímetros em um dos quatro pontos de sustentação do veículo).
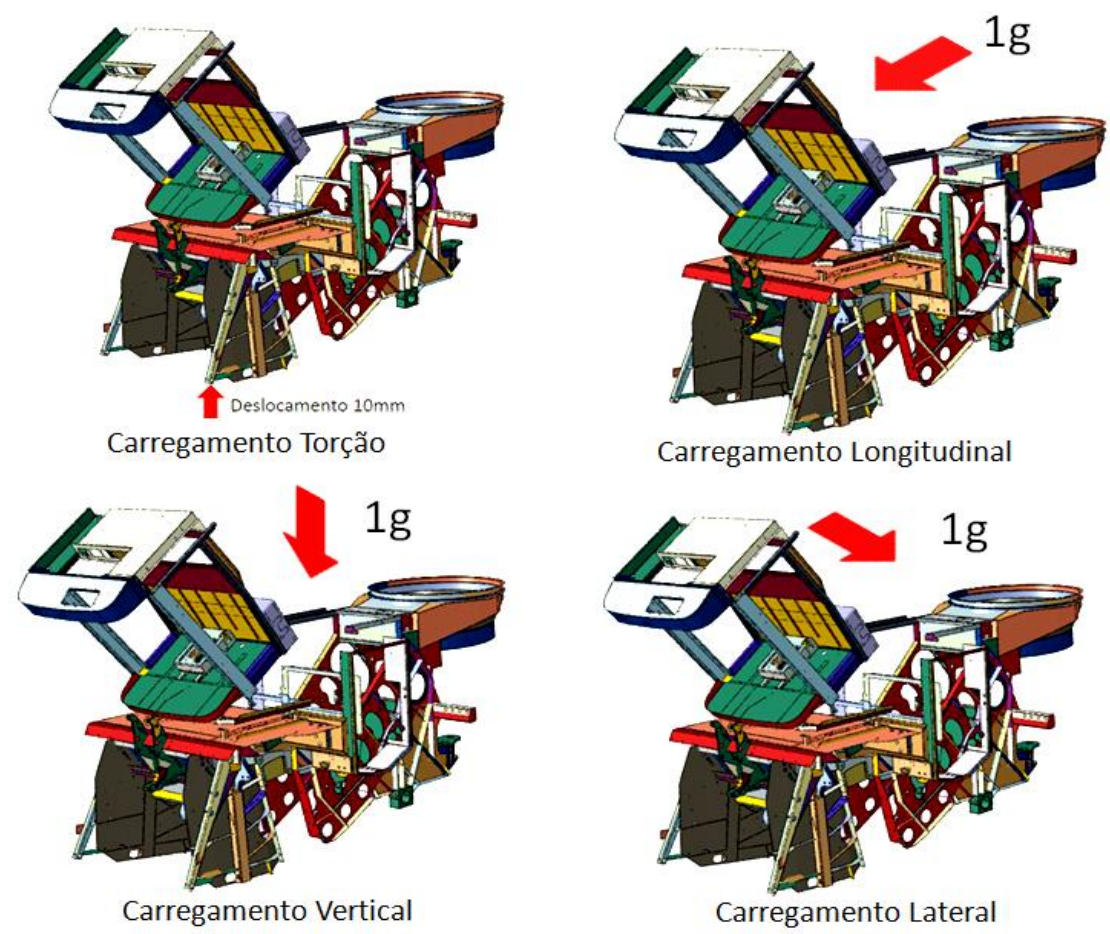

Figura 3: Casos de carregamento. 
Os resultados das análises mostraram inicialmente que todos os pontos de falha foram representados predominantemente no carregamento de torção.

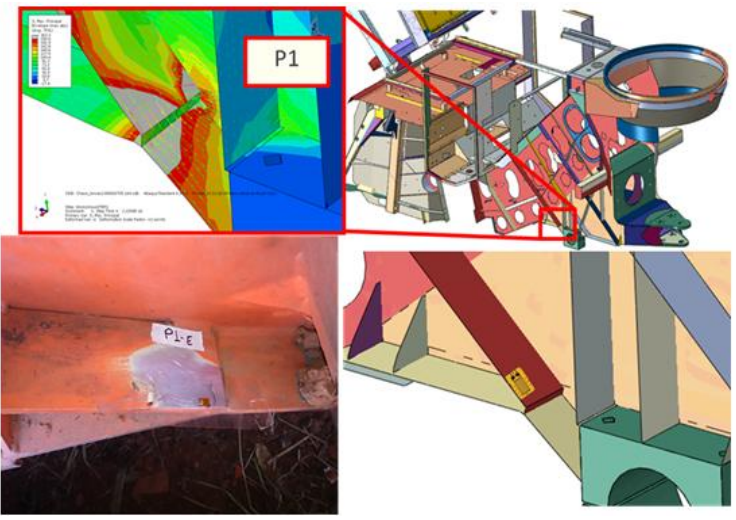

Figura 4: Ponto 1.

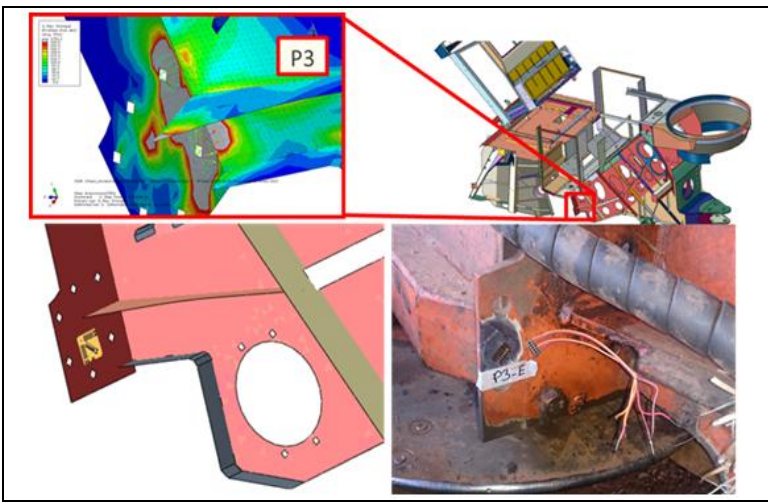

Figura 6: Ponto 3.

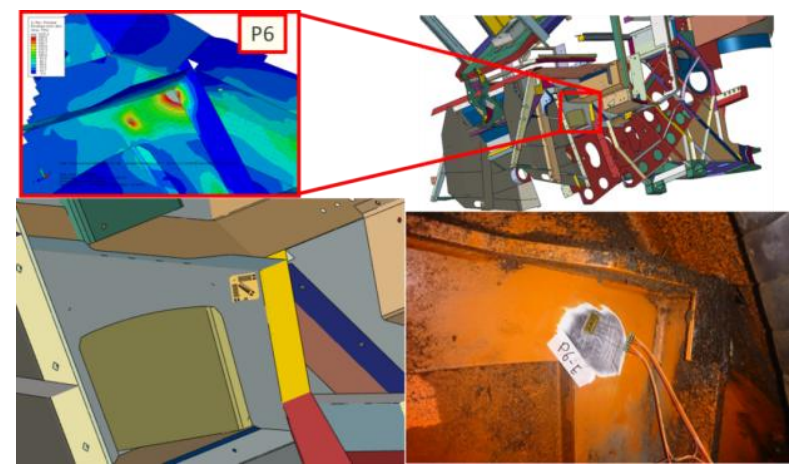

Figura 8: Ponto 6.

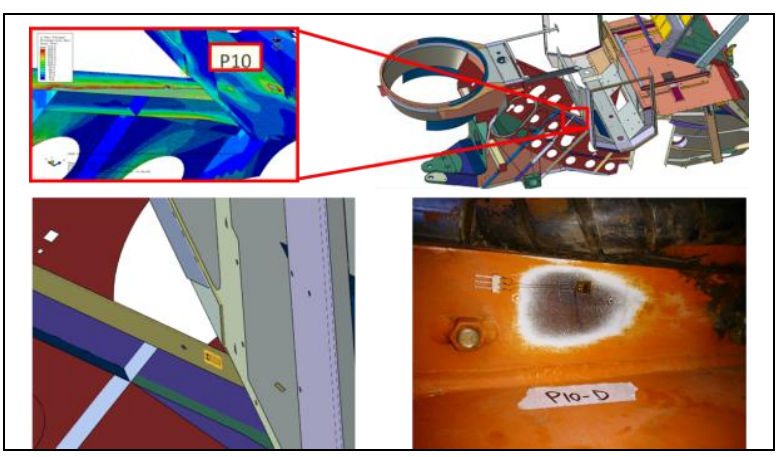

Figura 10: Ponto 10.

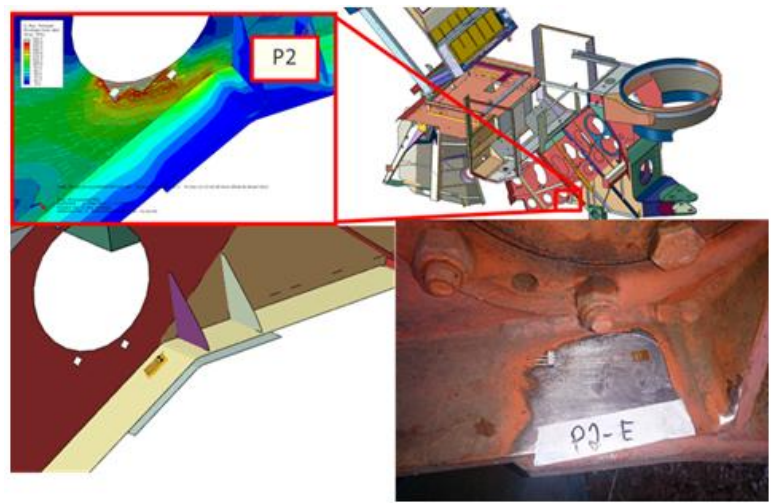

Figura 5: Ponto 2.

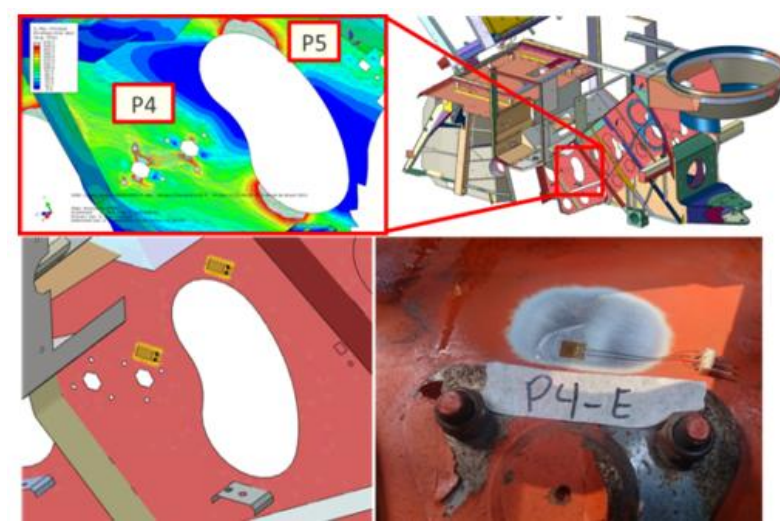

Figura 7: Ponto 4 e 5.

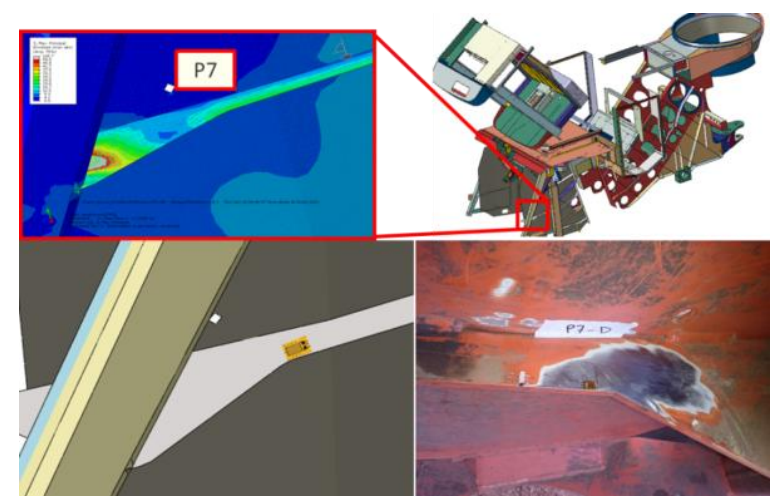

Figura 9: Ponto 7.

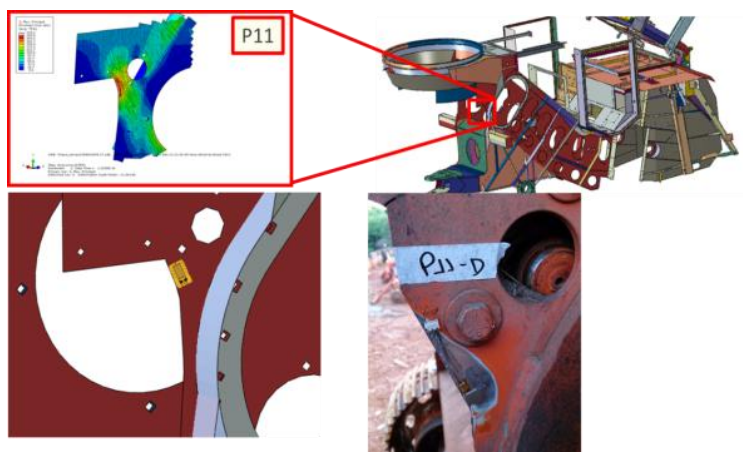

Figura 11: Ponto 11. 
Os dados coletados são analisados e a contagem de ciclos é realizada através da ferramenta Rain Flow que identifica padrões de ciclos de histerese (fadiga) em um sinal randômico, a Figura 12 mostra esse processo.

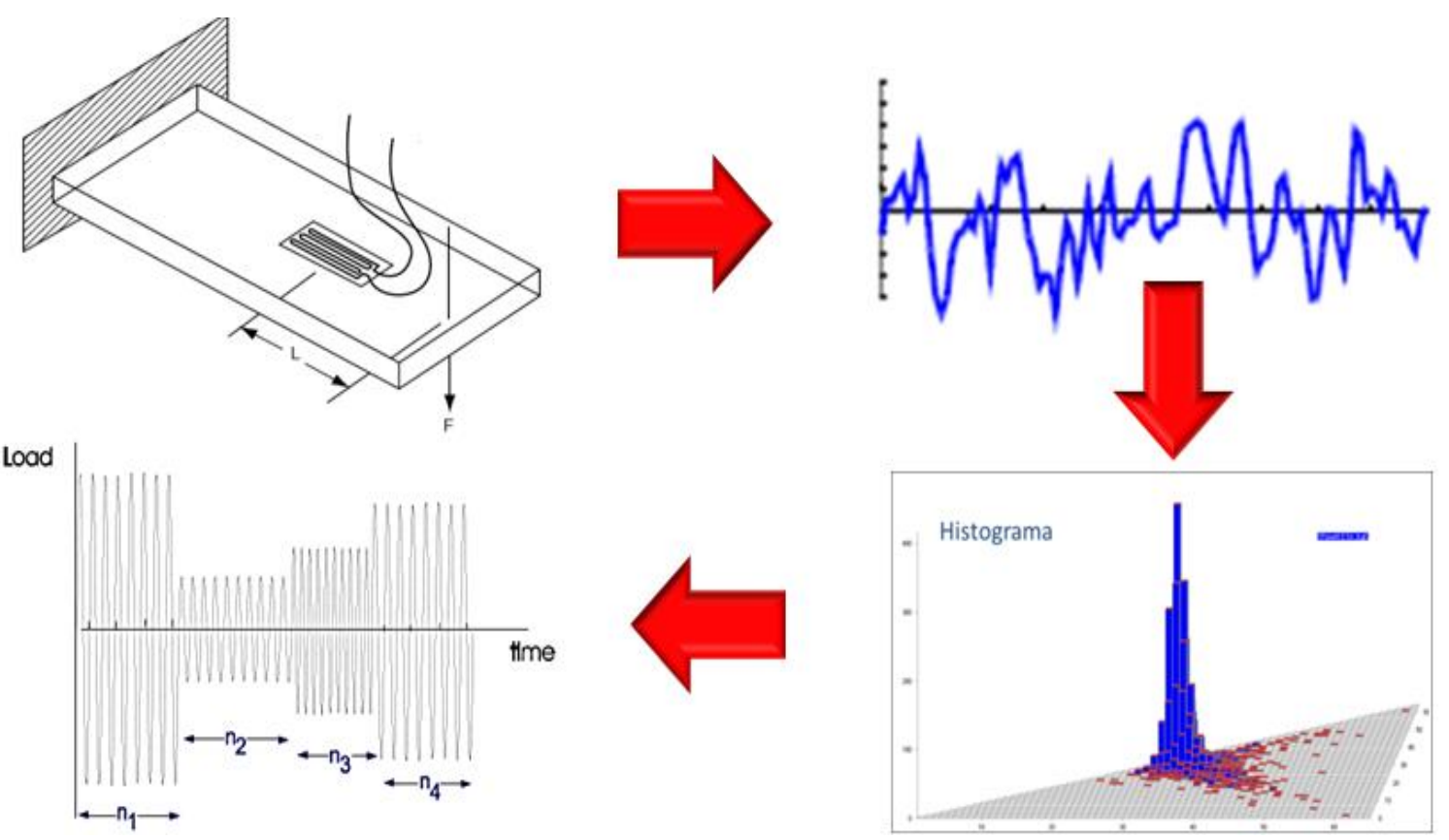

Figura 12: Processo de contagem de ciclos RainFlow.

Após a contabilização de ciclos o dano na estrutura foi calculado. A Tabela 1 mostra em vermelho o dano gerado na estrutura. Esse dano reflete a vida em campo para a maioria dos pontos.

Tabela 1: Dano nos pontos instrumentados.

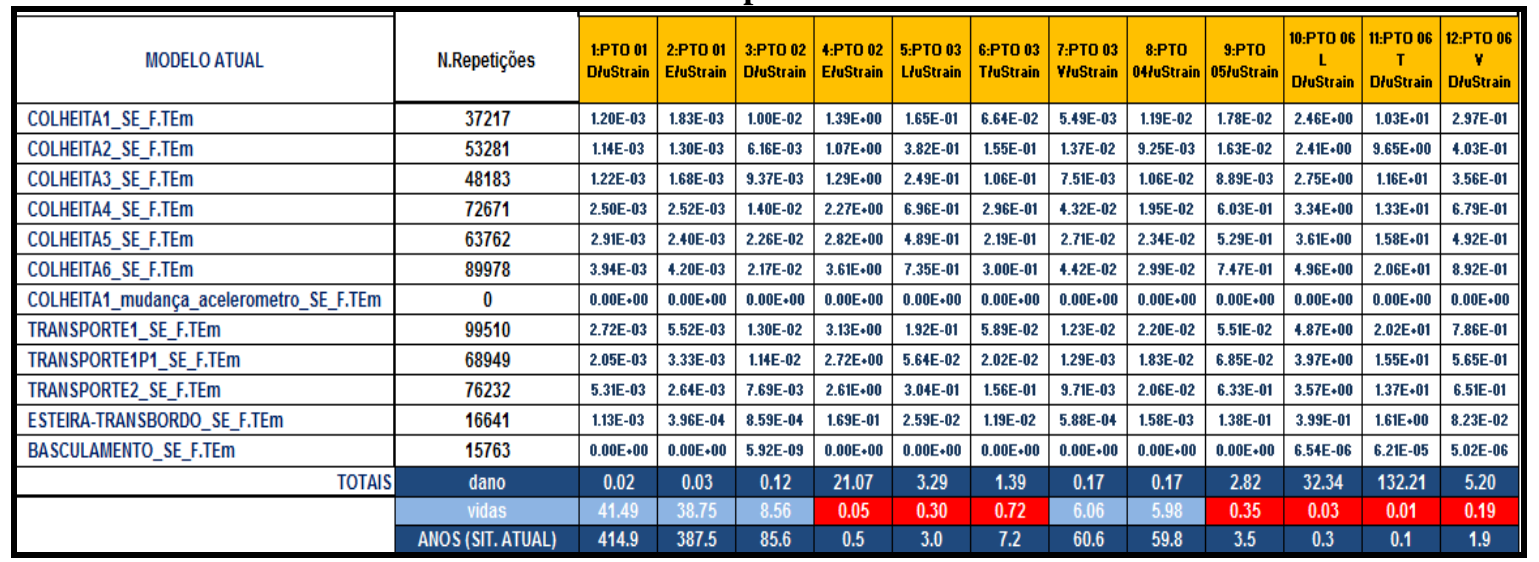

\section{MODELO DE ELEMENTOS FINITOS}

Com os dados de campo foi possível calibrar um valor de carregamento de torção que representa os pontos de falha. A Figura 13 mostra a força calibrada. 


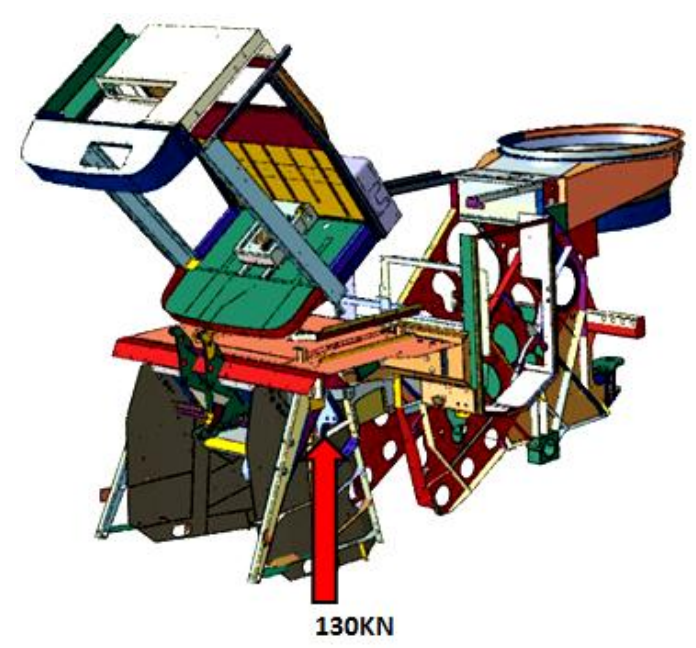

Figura 13: Carregamento de torção.

A Figura 14 mostra o resultado de tensão para o critério estabelecido de $130 \mathrm{MPa}$ para solda e 160MPa para chapa plana. As regiões em cinza estão acima do critério.
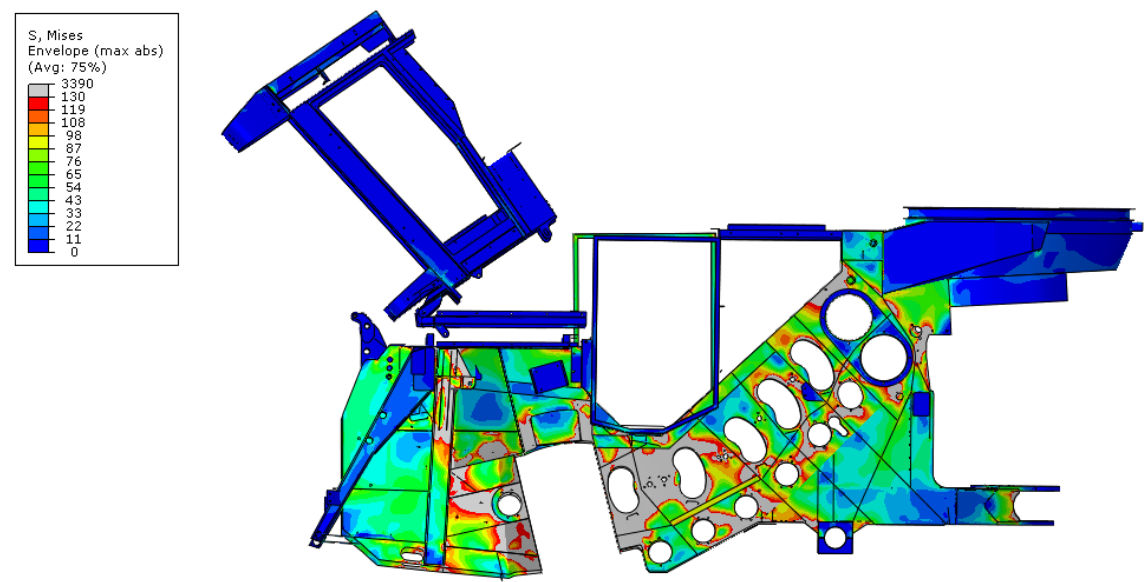

Figura 14: Resultado chassi base-line.

Após uma série de iterações de melhorias em parceria com a engenharia de produto obteve-se uma configuração de chassi que virtualmente atende o critério de tensão. A Figura 15 uma visão geral do chassi otimizado.

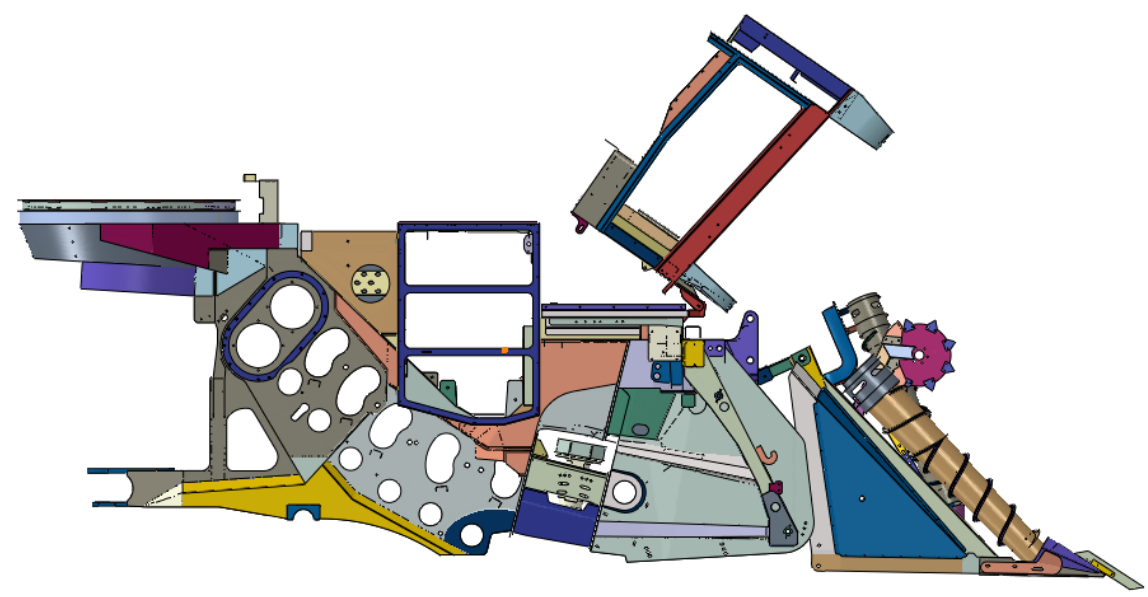

Figura 15: Estrutura Otimizada 
A Erro! Fonte de referência não encontrada. mostra o resultado de tensão do modelo otimizado.

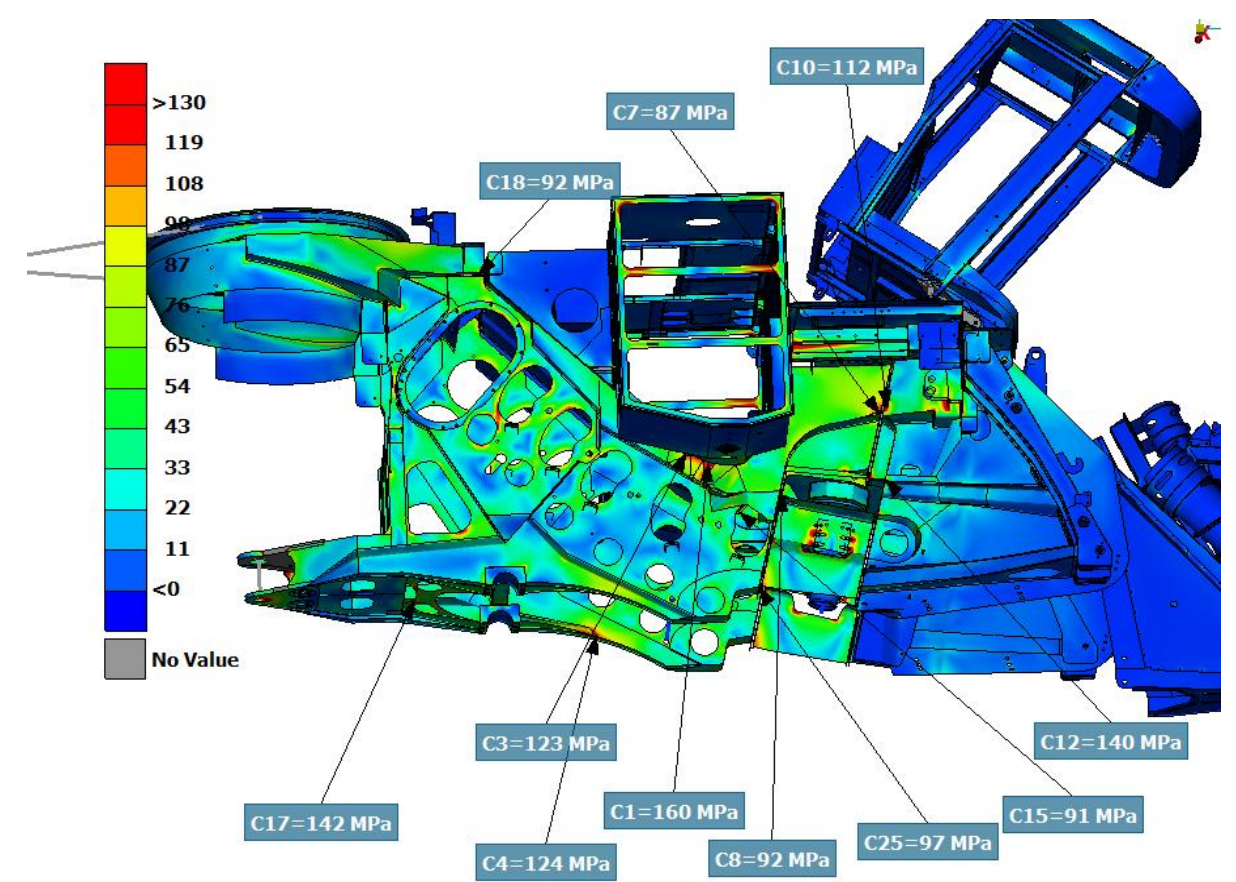

Figura 16: Resultado de tensão modelo otimizado.

A Tabela 2 mostra o resultado de tensão do modelo otimizado. Sendo que a última coluna mostra a margem de segurança em relação ao critério.

Tabela 2: Tensão modelo otimizado.

\begin{tabular}{|c|c|c|c|c|c|c|}
\hline & & \multirow{2}{*}{$\begin{array}{c}\text { FEA STRESS } \\
{[1 \mathrm{~g}]}\end{array}$} & \multirow{2}{*}{$\begin{array}{c}\text { LOAD }[g] \\
1.00 \\
\end{array}$} & \multicolumn{2}{|c|}{ MATERIAL FATIGUE CURVE } & \multirow{2}{*}{$\begin{array}{l}\text { SAFETY } \\
\text { MARGIN }\end{array}$} \\
\hline & & & & LIMIT & MATERIAL K & \\
\hline POINT & MATERIAL & & STRESS [Mpa] & [Mpa] & & \\
\hline C1 & PLAIN & 160 & 160 & 160 & 5 & $0 \%$ \\
\hline C2 & PLAIN & 147 & 147 & 160 & 5 & $9 \%$ \\
\hline C3 & $\mathrm{CC} 2$ & 123 & 123 & 130 & 3 & $5 \%$ \\
\hline C4 & $\mathrm{CC} 2$ & 124 & 124 & 130 & 3 & $5 \%$ \\
\hline C5 & $\mathrm{CC} 2$ & 122 & 122 & 130 & 3 & $7 \%$ \\
\hline C6 & $\mathrm{CC} 2$ & 85 & 85 & 130 & 3 & $54 \%$ \\
\hline C7 & $\mathrm{CC} 2$ & 87 & 87 & 130 & 3 & $50 \%$ \\
\hline C8 & $\mathrm{CC} 2$ & 92 & 92 & 130 & 3 & $41 \%$ \\
\hline C9 & $\mathrm{CC} 2$ & 72 & 72 & 130 & 3 & $80 \%$ \\
\hline C10 & $\mathrm{CC} 2$ & 112 & 112 & 130 & 3 & $16 \%$ \\
\hline C11 & $\mathrm{CC} 2$ & 81 & 81 & 130 & 3 & $61 \%$ \\
\hline C12 & PLAIN & 140 & 140 & 160 & 5 & $14 \%$ \\
\hline C13 & PLAIN & 149 & 149 & 160 & 5 & $8 \%$ \\
\hline C14 & $\mathrm{CC} 2$ & 124 & 124 & 130 & 3 & $5 \%$ \\
\hline C15 & $\mathrm{CC} 2$ & 91 & 91 & 130 & 3 & $43 \%$ \\
\hline C16 & PLAIN & 136 & 136 & 160 & 5 & $17 \%$ \\
\hline C17 & PLAIN & 142 & 142 & 160 & 5 & $12 \%$ \\
\hline C18 & $\mathrm{CC} 2$ & 92 & 92 & 130 & 3 & $41 \%$ \\
\hline C19 & $\mathrm{CC} 2$ & 104 & 104 & 130 & 3 & $25 \%$ \\
\hline $\mathrm{C} 20$ & $\mathrm{CC} 2$ & 127 & 127 & 130 & 3 & $2 \%$ \\
\hline C21 & $\mathrm{CC} 2$ & 109 & 109 & 130 & 3 & $19 \%$ \\
\hline $\mathrm{C} 22$ & $\mathrm{CC} 2$ & 110 & 110 & 130 & 3 & $18 \%$ \\
\hline C23 & $\mathrm{CC} 2$ & 100 & 100 & 130 & 3 & $30 \%$ \\
\hline C24 & $\mathrm{CC} 2$ & 118 & 118 & 130 & 3 & $11 \%$ \\
\hline $\mathrm{C} 25$ & $\mathrm{CC} 2$ & 97 & 97 & 130 & 3 & $34 \%$ \\
\hline
\end{tabular}




\section{TESTE ACELERADO EM LABORATÓRIO}

A fim de validar as hipóteses de cálculo e prever alguma outra falha antes da colhedora ir a campo um teste acelerado em laboratório foi elaborado. Porém antes de testar o chassi otimizado foi realizado um teste no chassi base line a fim de reproduzir as falhas de campo.

A Figura 17 mostra o chassi base line sendo ensaiado. O teste acelerado foi realizado aumentando-se a amplitude do carregamento de acordo com a regra de Miner utilizando curvas de fadiga obtidas com o teste de campo associado com o histórico de falha.
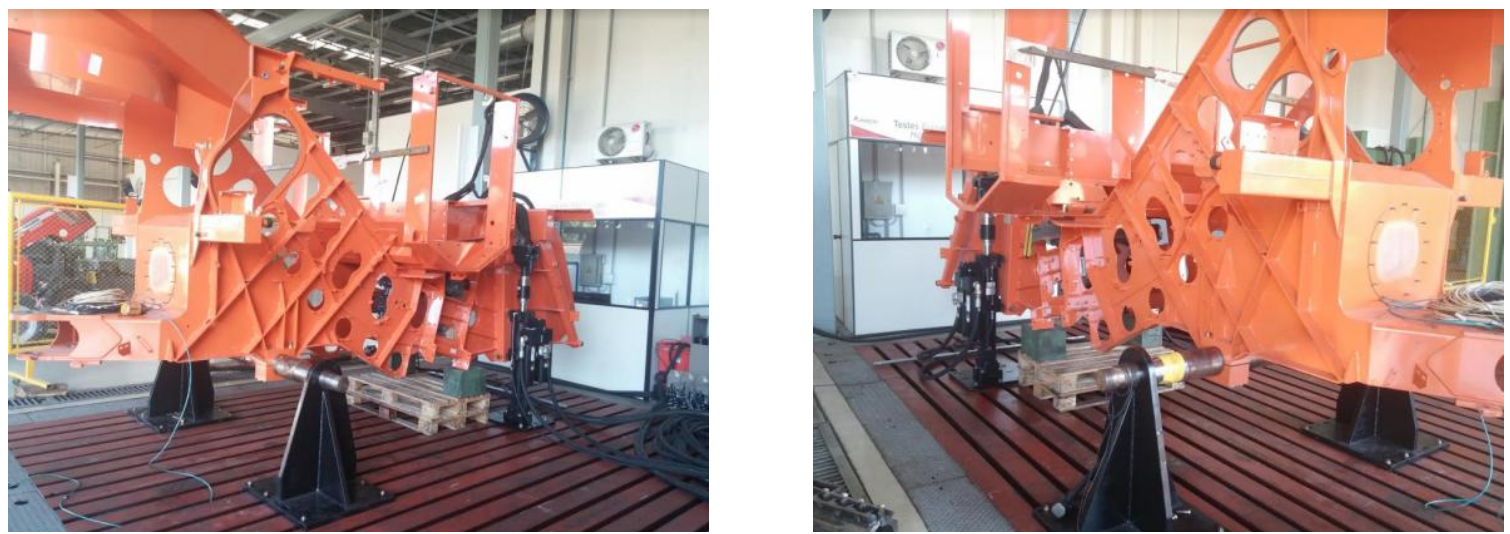

Figura 17: Ensaio chassi base line.

A Figura 18 mostra as trincas que surgiram em laboratório.
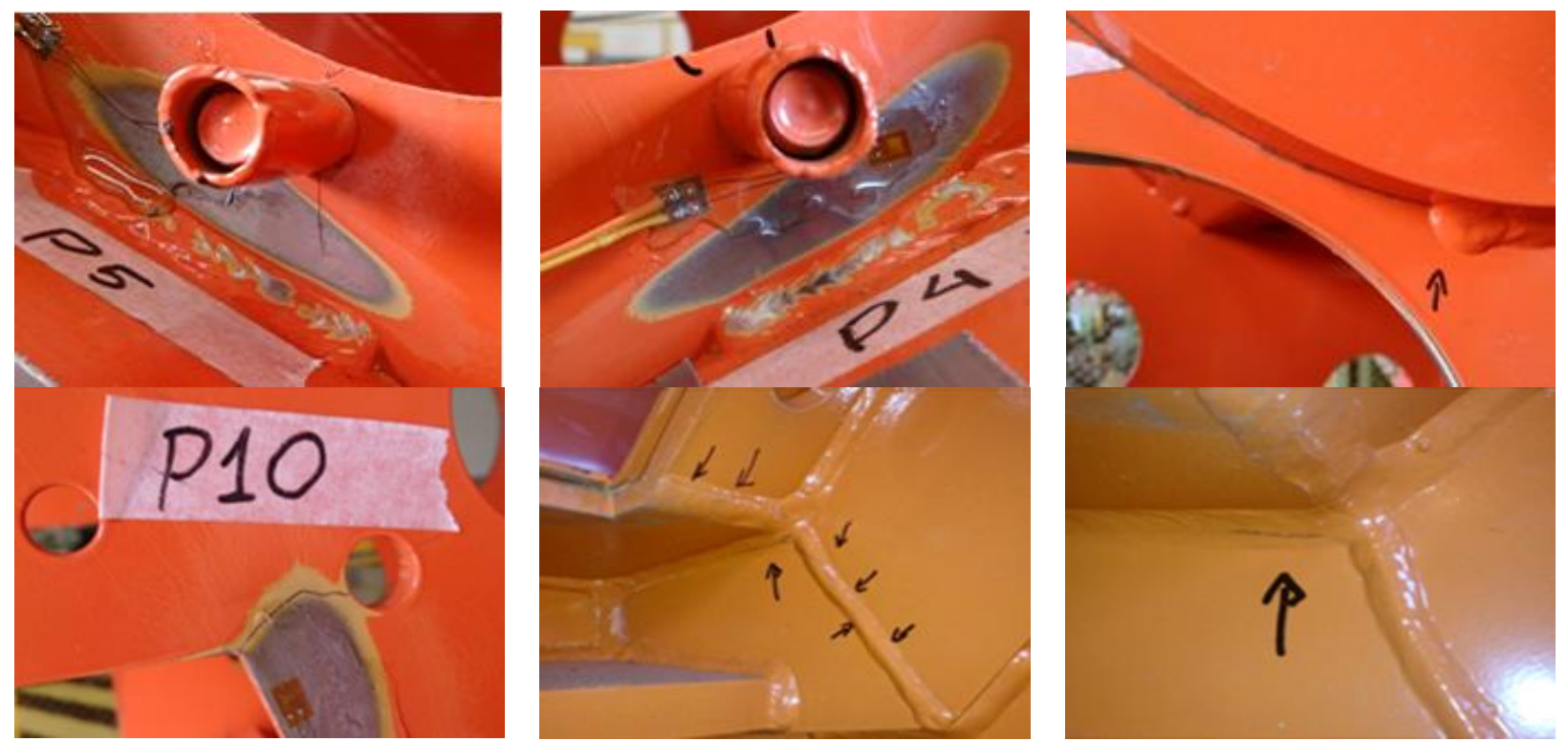

Figura 18: Trincas chassi base line.

Seguindo o mesmo procedimento do teste mostrado anteriormente um novo protótipo de chassi foi testado. $\mathrm{O}$ ensaio seria considerado se o teste durasse 50 horas com carregamento igual ao do teste anterior com frequência de $0,5 \mathrm{~Hz}$. 


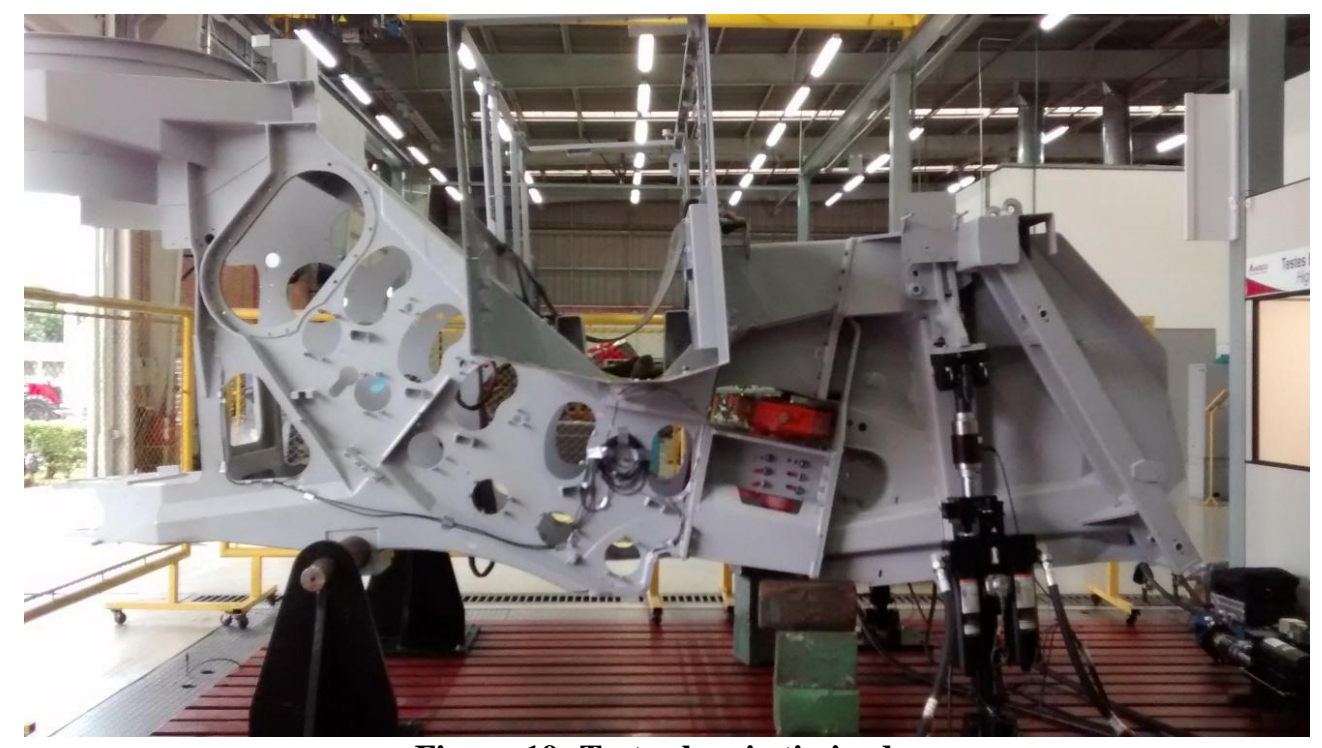

Figura 19: Teste chassi otimizado.

Mesmo com todos os procedimentos realizados anteriormente duas falhas surgiram durante o teste em laboratório.

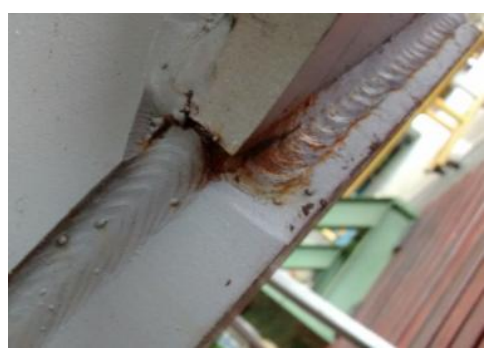

1

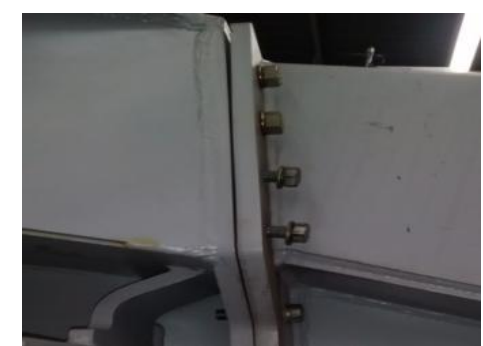

2

Figura 20: Falhas chassi otimizado.

A falha número 1 surgiu por questões de má qualidade de solda como mostrado na onde mostra a diferença entre os cordões de solda em lados simétricos. Já a falha 2 nos parafusos que une duas partes do chassi houve um erro no grau dos parafusos.

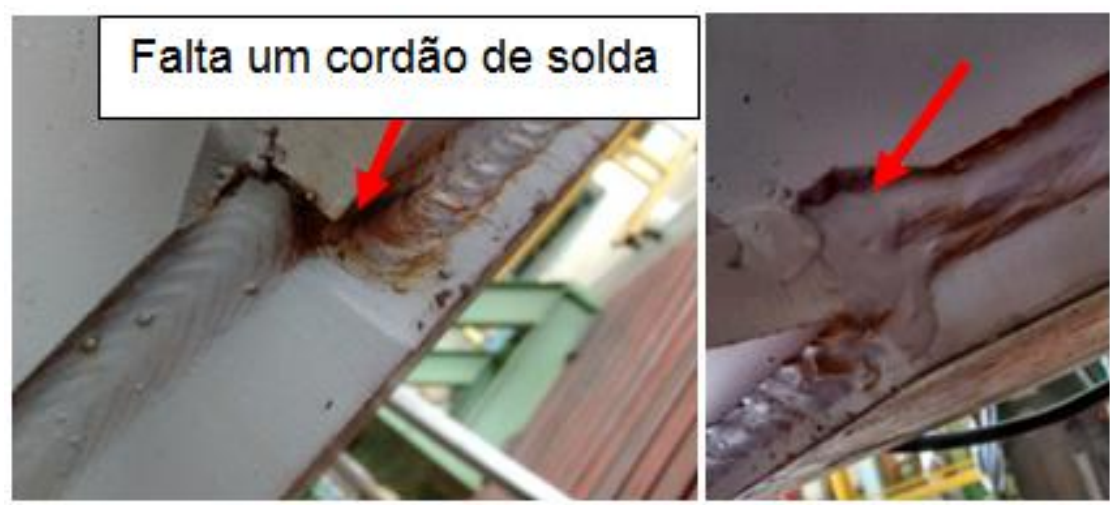

Figura 21: Diferença entre solda e lados simétricos. 


\title{
4. TESTE DE VALIDAÇÃ̃
}

Os pontos críticos da simulação foram instrumentados no protótipo de campo.

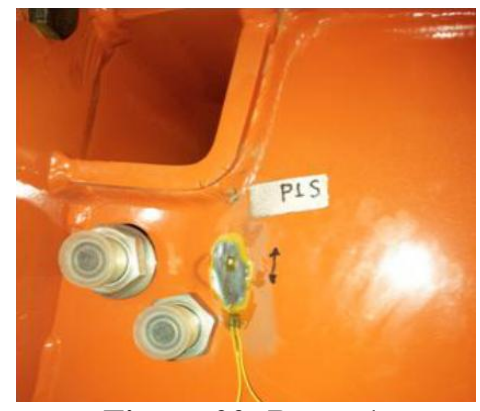

Figura 22: Ponto 1.

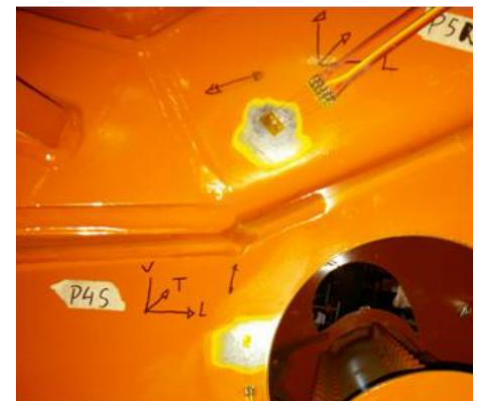

Figura 25: Ponto 4 e 5.

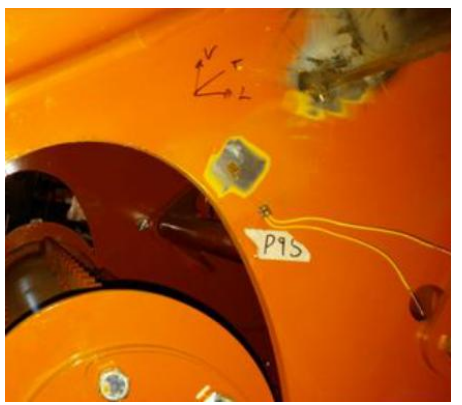

Figura 28: Ponto 9.

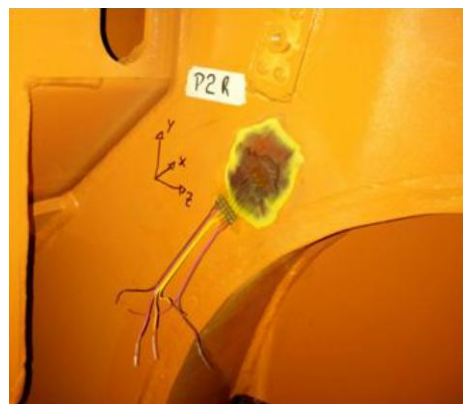

Figura 23: Ponto 2.

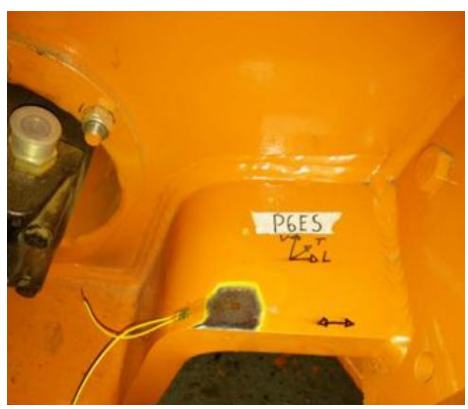

Figura 26: Ponto 6.

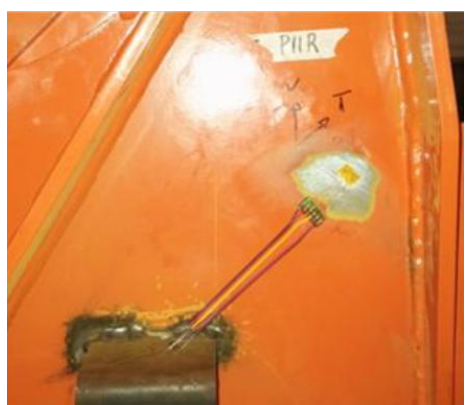

Figura 29: Ponto 11.

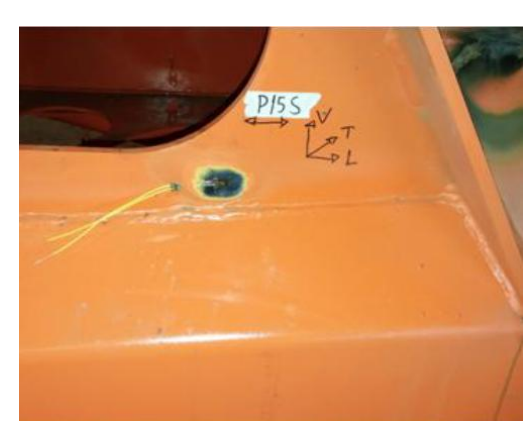

Figura 31: Ponto 15.

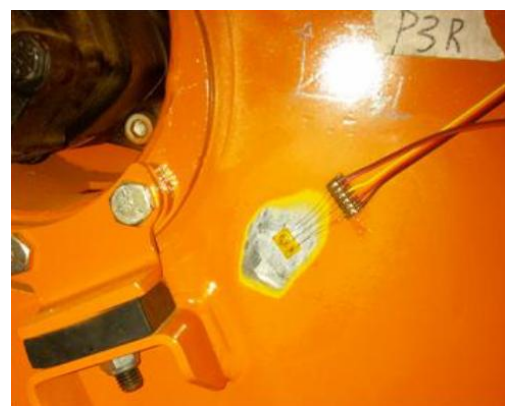

Figura 24: Ponto 3.

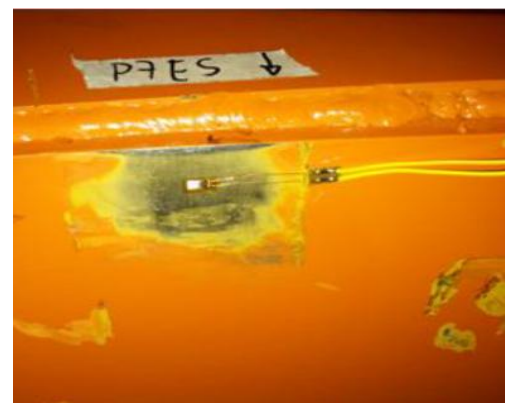

Figura 27: Ponto 7.

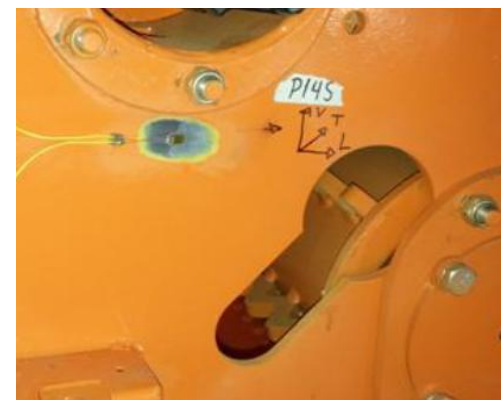

Figura 30: Ponto 14.

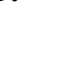

A Tabela 3 mostra os resultados de dano acumulado dos pontos instrumentados. 
Tabela 3: Resultado de dano acumulado.

\begin{tabular}{|c|c|c|c|c|c|c|c|c|c|c|c|c|}
\hline & |ATER| & LD & OLD & SOLDA & SOLDA & SOLDA & SOLD & SOLDA & HAPA & OLDA & OLDA & OLDA \\
\hline MODELO ATUAL & N.Repetiçöes & s01 & R02_V & R02_T & R02_L & R03_V & R03_T & R03_L & S04 & S05D & R05E_V & R05E_T \\
\hline COLHEITA1 & 3773 & $8.54 \mathrm{E}-02$ & $8.26 \mathrm{E}-03$ & $4.02 \mathrm{E}-03$ & $4.65 \mathrm{E}-04$ & $1.33 \mathrm{E}-02$ & $3.48 \mathrm{E}-02$ & $1.99 \mathrm{E}-03$ & $1.64 \mathrm{E}-04$ & $6.35 \mathrm{E}-04$ & $8.89 \mathrm{E}-04$ & $4.73 \mathrm{E}-03$ \\
\hline COLHEITA2 & 4427 & $9.69 \mathrm{E}-02$ & $9.12 \mathrm{E}-03$ & $5.11 \mathrm{E}-03$ & 5.34E-04 & $1.30 \mathrm{E}-02$ & $3.54 \mathrm{E}-02$ & $2.32 \mathrm{E}-03$ & $1.89 \mathrm{E}-04$ & $6.96 \mathrm{E}-04$ & $1.02 \mathrm{E}-03$ & $5.23 \mathrm{E}-03$ \\
\hline COLHEITA3 & 5429 & 1.25E-01 & 1.22E-02 & $5.77 \mathrm{E}-03$ & $8.64 \mathrm{E}-04$ & $1.34 \mathrm{E}-02$ & $3.81 \mathrm{E}-02$ & $2.47 \mathrm{E}-03$ & $2.42 \mathrm{E}-04$ & $7.50 \mathrm{E}-04$ & $1.31 \mathrm{E}-03$ & $6.55 \mathrm{E}-03$ \\
\hline TRANSPORTE & 8264 & $1.50 \mathrm{E}-01$ & $1.40 \mathrm{E}-02$ & $7.34 \mathrm{E}-03$ & $7.26 \mathrm{E}-03$ & $1.71 \mathrm{E}-02$ & $4.92 \mathrm{E}-02$ & $2.22 \mathrm{E}-03$ & $2.70 \mathrm{E}-04$ & $9.98 \mathrm{E}-04$ & 1.46E-03 & $9.76 \mathrm{E}-03$ \\
\hline \multirow[t]{3}{*}{ TOTAIS } & dano & 0.46 & 0.04 & 0.02 & 0.01 & 0.06 & 0.16 & 0.01 & 0.00 & 0.00 & 0.00 & 0.03 \\
\hline & vidas & 2.2 & 23.0 & 45.0 & 109.6 & 17.6 & 6.3 & 111.2 & 1155.0 & 324.8 & 213.6 & 38.1 \\
\hline & ANOS & 10.9 & 114.8 & 224.8 & 548.0 & 88.1 & 31.7 & 555.9 & 5774.8 & 1624.0 & 1068.1 & 190.3 \\
\hline
\end{tabular}

\begin{tabular}{|c|c|c|c|c|c|c|c|c|c|c|c|}
\hline & MATERIAL & SOLDA & SOLDA & SOLDA & SOLDA & SOLDA & SOLDA & SOLDA & SOLDA & CHAPA & SOLDA \\
\hline MODELO ATUAL & N.Repetiçöes & S06D & S06E & S07D & S07E & S09 & R11_V & R11_T & R11_L & s14 & s15 \\
\hline COLHEITA1 & 3773 & $5.63 \mathrm{E}-02$ & $6.42 \mathrm{E}-02$ & $3.29 \mathrm{E}-03$ & $8.27 \mathrm{E}-02$ & $1.01 \mathrm{E}-03$ & 9.30E-04 & $3.62 \mathrm{E}-02$ & $2.91 \mathrm{E}-02$ & $8.21 \mathrm{E}-02$ & $6.03 \mathrm{E}-04$ \\
\hline COLHEITA2 & 4427 & $6.36 \mathrm{E}-02$ & $6.67 \mathrm{E}-02$ & $3.81 \mathrm{E}-03$ & $9.81 \mathrm{E}-02$ & 1.12E-03 & $1.28 \mathrm{E}-03$ & 5.33E-02 & $4.28 \mathrm{E}-02$ & $1.29 \mathrm{E}-01$ & $6-49 \mathrm{E}-04$ \\
\hline COLHEITA3 & 5429 & $8.22 \mathrm{E}-02$ & $8.71 \mathrm{E}-02$ & $4.06 \mathrm{E}-03$ & 1.18E-01 & 1.13E-03 & $1.80 \mathrm{E}-03$ & $6.87 \mathrm{E}-02$ & $5.99 \mathrm{E}-02$ & 1.83E-01 & $4.61 \mathrm{E}-04$ \\
\hline TRANSPORTE & 8264 & $9.86 \mathrm{E}-02$ & 1.03E-01 & $3.64 \mathrm{E}-03$ & 1.45E-01 & $1.87 \mathrm{E}-03$ & $1.13 \mathrm{E}-03$ & $5.30 \mathrm{E}-02$ & $4.20 \mathrm{E}-02$ & $1.25 \mathrm{E}-01$ & $3.41 \mathrm{E}-04$ \\
\hline TOTAIS & dano & 0.30 & 0.32 & 0.01 & 0.44 & 0.01 & 0.01 & 0.21 & 0.17 & 0.52 & 0.00 \\
\hline & vidas & 3.3 & 3.1 & 67.6 & 2.3 & 194.4 & 194.7 & 4.7 & 5.8 & 1.9 & 486.9 \\
\hline & ANOS & 16.6 & 15.6 & 337.8 & 11.3 & 972.1 & 973.7 & 23.7 & 28.8 & 9.6 & 2434.6 \\
\hline
\end{tabular}

\section{CONCLUSÃO}

A metodologia de trabalho se mostrou eficiente para a elaboração e desenvolvimento de um novo produto.

A medição inicial em um produto base line é a maneira mais prática para se levantar os esforços de campo. A simulação de elementos finitos no auxílio da direção de colagem dos extensômetros se torna interessante não só para diminuir o número de canais de medição, mas principalmente dar uma ideia inicial do comportamento global da estrutura.

Com o resultado das medições consegue-se calcular o dano na estrutura e também com o auxílio do modelo de elementos finitos realizar uma calibração que torna a estrutura um dispositivo de medição de carregas.

A etapa de otimização virtual é bastante trabalhosa e passa por uma série de iterações que depende muito do bom relacionamento entre engenharia de produto e engenharia de cálculo. O chassi teve um aumento de peso de $30 \%$ porém sua expectativa de vida passou de uma média de $1200 \mathrm{~h}$ para 15000 horas.

Os ensaios acelerados de laboratório são de extrema importância para validação do protótipo. De maneira rápida e econômica é possível ter uma excelente perspectiva do desempenho do produto em campo. Esse ensaio trouxe uma redução de 15000 horas para 50 horas. Algumas falhas ocorreram nessa fase, porém como mencionado na Seção 3 as falhas foram de manufatura e não de projeto.

O ensaio de campo confirmou que os danos em cada ponto medido estão dentro do limite de estimado e simulação.

Assim conclui-se que a metodologia aplicada é robusta o suficiente para o desenvolvimento de produtos e com o passar do tempo o conhecimento sobre o produto se torna maior e a tendência é que o desenvolvimento se trone cada vez mais aperfeiçoada. 


\section{REFERÊNCIAS}

[1] LEE, Y.; PAN, J.; HATHAWAY, R.; BARKEY, M. Fatigue Testing and Analysis. Elsevier, Press, 2005. 402pp.

[2] LITTLE, R.E. \& EKVALL, J.C. Statistical Analysis of Fatigue Data. American Society for Testing and Materials, ASTM, 1979. 141PP.

[3] WONG, J.T. Theory of Ground Vehicles. 3 ed. John Wiley, 2001. 528pp. 